
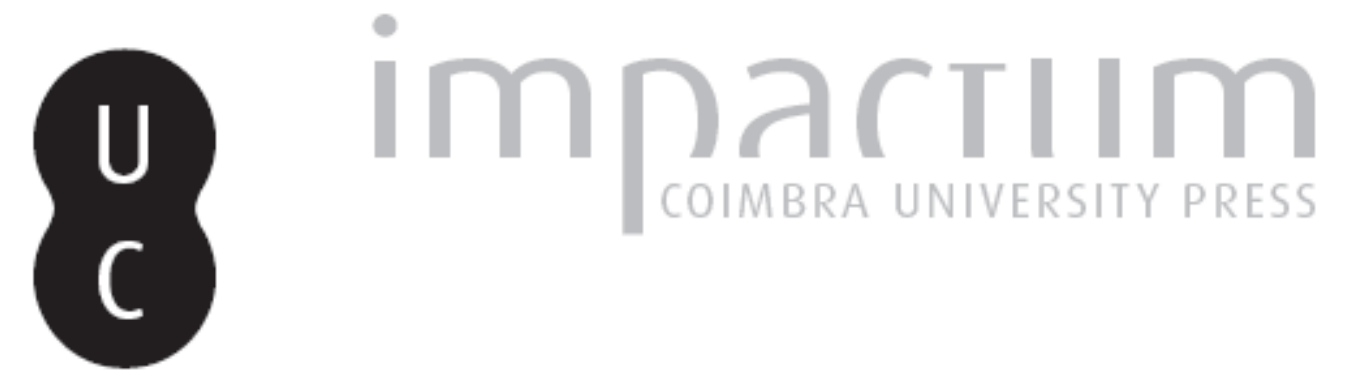

\title{
Obesidade ante et post cirurgia
}

Autor(es): $\quad$ Silva, Marlene; Padez, Cristina

Publicado por: Imprensa da Universidade de Coimbra

URL persistente:

URI:http://hdl.handle.net/10316.2/39079

DOI:

DOI:http://dx.doi.org/10.14195/2182-7982_31_6

Accessed : $\quad$ 26-Apr-2023 14:18:38

A navegação consulta e descarregamento dos títulos inseridos nas Bibliotecas Digitais UC Digitalis, UC Pombalina e UC Impactum, pressupõem a aceitação plena e sem reservas dos Termos e Condições de Uso destas Bibliotecas Digitais, disponíveis em https://digitalis.uc.pt/pt-pt/termos.

Conforme exposto nos referidos Termos e Condições de Uso, o descarregamento de títulos de acesso restrito requer uma licença válida de autorização devendo o utilizador aceder ao(s) documento(s) a partir de um endereço de IP da instituição detentora da supramencionada licença.

Ao utilizador é apenas permitido o descarregamento para uso pessoal, pelo que o emprego do(s) título(s) descarregado(s) para outro fim, designadamente comercial, carece de autorização do respetivo autor ou editor da obra.

Na medida em que todas as obras da UC Digitalis se encontram protegidas pelo Código do Direito de Autor e Direitos Conexos e demais legislação aplicável, toda a cópia, parcial ou total, deste documento, nos casos em que é legalmente admitida, deverá conter ou fazer-se acompanhar por este aviso.

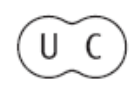




$$
\bar{A}
$$




\title{
Obesidade ante et post cirurgia
}

\author{
Marlene Silva', Cristina Padez ${ }^{1,2}$ \\ cpadez@antrop.uc.pt \\ DOI: http://dx.doi.org/10.14195/2182-7982_31_6
}

Resumo $O$ presente estudo tem por objetivo analisar a perda de peso após a intervenção cirúrgica em indivíduos obesos, verificar o seu grau de efetividade e tentar perceber a razão pela qual alguns pacientes conseguem perder peso e mantê-lo e outros não. Foi ainda avaliado o impacto das alterações associadas ao estilo de vida e hábitos alimentares após a cirurgia. Os dados foram recolhidos no Serviço de Cirurgia do Hospital de São Sebastião, localizado em Santa Maria da Feira. Foram examinados processos de pacientes operados há mais de três anos, dos quais 82 apresentavam processos válidos e completos. Destes, 67 eram mulheres e 15 eram homens. Perderam
Abstract The study aim is to analyze the weight loss after surgery in obese individuals, check the degree of effectiveness and try to understand why some patients can lose weight and maintain it and others are not able to lose and preserve weight loss. The impact of the lifestyle and diet were also evaluated after the surgery.

The data were collected in the surgery service of Hospital de São Sebastião, located at Santa Maria da Feira. Processes from patients that underwent surgery more than three years before were examined, from which 82 had valid and complete processes: 67 were women and 15 were

\footnotetext{
1 Centro de Investigação em Antropologia e Saúde, Universidade de Coimbra, Coimbra, Portugal.

2 Departamento de Ciências da Vida, Universidade de Coimbra, Coimbra, Portugal.
} 
peso e estabilizaram $81,3 \%$ dos pacientes, os restantes $18,7 \%$, após a perda de peso, aumentaram mais de cinco quilos. A prática de exercício físico esteve associada a um maior decréscimo do índice da massa corporal (IMC), tanto para os que praticaram mais de 30 minutos por dia, como para os que praticaram menos, em comparação com aqueles que não praticaram qualquer atividade física. Os melhores resultados foram encontrados entre os pacientes que simultaneamente cumpriram a dieta e fizeram mais de 30 minutos de exercício físico por dia. Daqui se deduz que os níveis de sucesso mais elevados ocorrem entre os que cumprem a dieta alimentar e os que aderem à prática de exercício físico.

Palavras-chave: Obesidade; cirurgia bariátrica; dieta; atividade física; índice de massa corporal.

\section{Introdução}

\subsection{A evolução da obesidade}

Desde há algum tempo que a obesidade suscita grandes inquietudes, o que por sua vez tem provocado o aumento considerável de investigações nas mais diversas áreas científicas. $\mathrm{O}$ aumento da obesidade está relacionado com o sedentarismo, erros alimentares e com o próprio ritmo alterado da vida moderna, entre muitos outros fatores, como men. The patients who lost weight and stabilized were $81.3 \%$, and the remaining 18.7\%, after losing some weight, increased more than 10.4 pounds. The physical activity is associated to a greater decrease of the body mass index (BMI), both for those who practiced more than 30 minutes daily and for those who practised less time, compared to those who did not practice. The best results were found among the patients who met the diet requisites and practiced more than 30 minutes of daily physical activity. It follows that the higher levels of success occur among those complying with the diet and adhering to physical exercise.

Keywords: Obesity; bariatric surgery; diet; physical activity; body mass index.

os genéticos, metabólicos, hormonais e ambientais. Todavia, o contributo destes fatores não se encontra totalmente esclarecido. A abordagem antropológica da obesidade engloba uma dimensão evolutiva e cultural, que procura compreender como é que a predisposição para a obesidade nas sociedades atuais pode ter sido determinante ao longo da evolução humana enquanto caçadores-recolectores (Brown, 1998). Esta abordagem tenta ainda explicar a variação na prevalência da obesidade em diferentes sociedades, 
classes sociais e grupos étnicos (Brown, 1998). É importante compreender o nosso percurso evolutivo porque, de facto, durante a maior parte da história da humanidade a obesidade nunca foi um problema de saúde (Padez, 1999/2000). Apesar da qualidade adequada em termos de dieta, a maioria das sociedades de caçadores-recolectores esteve frequentemente sujeita a períodos de carência alimentar, o que terá constituído um forte agente de seleção natural na evolução biocultural humana (Cordain, 2002).

Atualmente é possível verificar uma manifesta preocupação relacionada com a prevalência da obesidade e os riscos que esta pode causar à saúde, uma vez que a obesidade aumenta a probabilidade de doenças como a diabetes mellitus tipo 2, dislipidemia (colesterol e triglicerídeos elevados), hipertensão arterial, doenças cardiovasculares, problemas respiratórios, apneia do sono, doença da vesícula biliar, doenças osteoarticulares e determinados cancros (Castelnuovo e Simpson, 2011). A OMS estima que mil milhões de adultos tenham excesso de peso e que mais de 300 milhões sejam obesos. No ano de 2014, mais de 1,9 mil milhões de adultos, com mais de 18 anos de idade, tinham excesso de peso. Destes, mais de 600 milhões eram obesos (OMS, 2015). Também segundo a OMS (2014), morrem anualmente pelo menos 2,8 milhões de pessoas como resultado do excesso de peso.
Mas qual a razão para as pessoas estarem cada vez mais obesas? Este aumento significativo de pessoas obesas surge devido à incompatibilidade entre as características biológicas do indivíduo e o ambiente moderno, que tem vindo a sofrer transformações de uma forma drástica (Power e Shulkin, 2009). Atualmente é visível o estilo de vida sedentário nas sociedades desenvolvidas, em que a disponibilidade abundante de alimentos faz com que haja um menor dispêndio de energia, levando a um desequilíbrio do balanço energético (Padez, 2002).

\subsection{Caracterização das diferentes épocas}

A obesidade não é exclusiva da era moderna, porém, a sua prevalência tem-se alterado consideravelmente nos últimos anos (Power e Shulkin, 2009). Evidências da obesidade humana remontam há mais de 20 mil anos com um achado arqueológico na Alemanha, a denominada Vénus de Willendorf. Não se sabe ao certo se tal obra de arte é uma verdadeira representação de um indivíduo, todavia, o nível de realismo detalhado na figura indica que o artista reproduzia uma mulher obesa (Power e Shulkin, 2009). A medicina grega considerava o excesso de gordura uma patologia, para a qual desenvolveu o conceito "polysarkia", que significa muita carne (Gilman, 2010). Hipócrates assumia 
uma linha de diferença entre a gordura aceitável e a excessiva, associando a primeira à vida e a segunda à morte (Gilman, 2010). No ensaio de Aristóteles, o tecido adiposo era visto como uma qualidade de preservação e os animais, incluindo os humanos, eram naturalmente húmidos e quentes, enquanto um corpo morto era caracterizado por ser seco e frio (Gilman, 2010). Contemporaneamente, é do conhecimento científico que o armazenamento de gordura é um processo adaptativo e que a gordura é essencial para a sobrevivência da espécie. Os humanos evoluíram de forma positiva relativamente ao armazenamento de gordura, por exemplo os bebés humanos são considerados os mais gordos de todos os mamíferos (Power e Shulkin, 2009). As virtudes do corpo clássico grego eram a força, a saúde e a beleza. O povo grego apreciava trabalhar tanto o intelecto como o corpo. Os primeiros romanos seguiram de perto esta linha condutora da medicina grega, caracterizando a obesidade como uma patologia (Gilman, 2010). Do século XVII ao século XIX, o sucesso do padrão de beleza era representado por indivíduos corpulentos. A arte ocidental da época caracterizava as figuras roliças, como se pode verificar no trabalho de Peter Paul Rubens, através da pintura a Vénus do espelho, um entre os inúmeros quadros do artista (Stearns, 2002). As mulheres eram constantemente elogiadas pelas suas formas robustas e voluptuosas, dado que neste período a robustez estava intrinsecamente relacionada com o sucesso da maternidade. Já para um cavalheiro, uma barriga saliente era motivo de orgulho, exibida com desembaraço. O aspeto corpulento estava indissociavelmente ligado à prosperidade, testemunhando o sucesso conseguido por determinado indivíduo. Os médicos salientavam a importância do peso contra o nervosismo, demonstrando como a magreza estava correlacionada com o descontentamento e com personalidades nervosas (Stearns, 2002). Daniel Lambert foi um caso singular de extrema obesidade no século XVIII em Inglaterra. Durante a sua vida, tornou-se particularmente famoso devido ao seu extraordinário peso. Aquando da sua morte, aos 39 anos, Daniel Lambert pesava 335 quilos, media 1,80 metros e tinha um perímetro abdominal de 284 centímetros (Power e Shulkin, 2009). Na sua juventude era um pouco robusto, contudo nada de exagerado, tendo sido considerado um excelente nadador. Relativamente à sua alimentação, esta era considerada moderada. Após a sua mudança para um trabalho de secretária, começou a passar a maior do tempo sentado (Power e Shulkin, 2009).

Como é possível constatar, as formas corpulentas são conhecidas há muito entre os seres humanos, o que leva a crer numa forte componente genética ou patológica (Power e Shulkin, 2009). Sinais de mudança começam a surgir no ano de 1890, quando os corpos redondos 
começam a dar espaço aos corpos mais magros. Assim, no ano de 1900, salvo algumas exceções de voluptuosidade, a imagem de magreza já se encontrava imposta e triunfava largamente.

Presentemente, a magreza é o protótipo mais desejado pelas mulheres dos países desenvolvidos, aspiração que ocorre num cenário onde é fácil tornar-se obeso. Nas sociedades desenvolvidas, a tendência global é o aumento de pessoas obesas nos grupos menos favorecidos, enquanto os sujeitos de maior estatuto socioeconómico são proporcionalmente mais magros (Brown, 1998).

Contemporaneamente, a existência de pessoas magras e insatisfeitas com o seu peso é de igual forma marcante, sendo que tal insatisfação pode levar à morte (Brown, 1998). Convém lembrar que as questões de gordura e magreza são muito mais do que questões médicas, biológicas e de desempenho individual. O aumento da prevalência de pessoas obesas nos países em desenvolvimento é surpreendente, podendo ser observada em indivíduos com melhores condições económicas. Porém, são vários os motivos associados a este rápido agravamento. Além da globalização, que torna o movimento de trocas mais veloz, existe outro especto que promove o aumento da obesidade nos países em desenvolvimento e está relacionado com questões culturais. A obesidade nestes países não é estigmatizada, aliás, é desejável em certas culturas mulheres com excesso de peso (Nour, 2010). A obesidade encontra-se associada à beleza, ao elevado estatuto socioeconómico e ao poder e qualidade na saúde. Pelo contrário, nos países desenvolvidos, a obesidade está inversamente relacionada com o estatuto socioeconómico elevado.

\subsection{Hábitos alimentares}

O impulso de comer é mais forte do que o impulso sexual (Richard, 1948 in Mintz, 2001)

"A alimentação é uma necessidade básica, um direito humano, assim como uma atividade cultural que envolve tabus, crenças, diferenças" (Pinheiro, 2005: 173). $O$ ato de alimentar não representa somente o facto de ingerir nutrientes relevantes para o funcionamento do corpo. Este desempenha um papel social influenciado pelo convívio, pela liberdade, pelas diferenças existentes e pelo domínio (Pinheiro, 2005). Pode dizer-se que os padrões alimentares de um grupo expressam uma identidade coletiva, assim como a posição na hierarquia e na organização social.

Nas últimas décadas, têm sido observadas mudanças nos hábitos alimentares nos mais diversos países, o que revela a complexidade dos modelos de consumo 
e fatores determinantes (Pinheiro, 2005). Para que os aspetos económicos, sociais, culturais e nutricionais da alimentação possam ser avaliados, é necessário adotar uma abordagem multidisciplinar e comparativa (Garcia-Closas et al., 2006). Tal abordagem permite compreender os mecanismos responsáveis pelas mudanças e consequências nos diferentes contextos socioeconómicos.

\subsection{Atividade física}

O movimento é uma forma de expressão do Homem

(Nunes, 2006)

Durante $95 \%$ a $99 \%$ da história da humanidade, as populações viveram com um padrão de subsistência de caçadores-recoletores, em que necessitavam de despender uma elevada quantidade de energia para conseguir alimentos para a sua subsistência (Padez, 2002). "O nível de sedentarismo das sociedades actuais deriva, entre outros factores, da evolução cultural da humanidade" (Padez, 2002:11). Presentemente, os alimentos estão disponíveis de forma abundante, não sendo necessário um grande dispêndio de energia para os obter, o que provoca um desequilíbrio no balanço energético (Padez, 2002).
Na realidade, o ser humano tem vindo a criar um mundo diferente, bem mais confortável, e é também verdade que estas mudanças trazem consigo novos desafios difíceis de superar. É compreensível que, depois de tempos complicados como a escravatura, o trabalho árduo de sol a sol até aos dias de hoje, o ser humano desfrute de mais tempo disponível para o repouso e para as atividades de lazer. Porém, este alargamento do lazer está cada vez menos preenchido com a prática de exercício físico (Nunes, 2006). As estruturas biológicas do ser humano estão preparadas e moldadas para o movimento e a atividade física (Nunes, 2006). Deste modo, a inatividade dos tempos modernos tem como consequência a perturbação do equilíbrio funcional dos indivíduos (Nunes, 2006). De acordo com Padez (2002: 16) "o peso, a composição corporal e o armazenamento de energia na forma de triglicerídeos no tecido adiposo são determinados pela interacção entre os factores genéticos, ambientais e psico-sociais". Todas estas influências atuam variando a equação do balanço energético, isto é, a proporção entre o consumo e o dispêndio de energia (Padez, 2002).

O estilo de vida das sociedades atuais é caracterizado por uma quase ausência de atividade física, sendo que o número de horas passadas a ver televisão parece ser um contributo importante para esta inatividade (Gortmaker et al., 1990 in Padez, 1999/2000). Outros fatores que se 
encontram relacionados com a obesidade são o baixo nível de instrução e um menor rendimento (Pories, 2008).

É evidente a elevada taxa de obesidade no sexo feminino em países como os Estados Unidos da América, Brasil e França, contrariamente ao que acontece em Portugal, onde os homens são os mais afetados (Carmo et al., 2008).

Nas sociedades atuais, as pessoas obesas experienciam elevados níveis de stresse, o que provavelmente está relacionado com uma forte representação social de que o obeso é uma pessoa dotada de pouca motivação, baixa autoestima e que ignora os padrões estéticos (Papelbaum et al., 2010).

A cirurgia bariátrica tem surgido como uma ferramenta necessária na perda de peso, sendo o impulso gerador de alterações fundamentais. O bypass é atualmente considerado uma prática promissora no tratamento da obesidade e da diabetes a longo prazo (Hussain et al., 2009). Porém, o paciente deve estar preparado para as grandes transformações que the serão exigidas, quer a nível de hábitos alimentares, quer ao nível da atividade física, já que estas são essenciais para atingir o sucesso desejado.

Ao nível das características psicossociais existe uma evolução na autoestima, na saúde relacionada com a qualidade de vida e na imagem corporal (Sarwer e Fabricatore, 2008). Assim como a qualidade de vida, a imagem corporal é uma construção multidimensional que tem sido associada de uma forma positiva ao IMC (Sarwer et al., 2010).

\section{Objectivos}

O objetivo do presente estudo é analisar a perda de peso após a intervenção cirúrgica em indivíduos obesos, verificar o seu grau de efetividade e tentar perceber a razão pela qual alguns pacientes conseguem perder peso e mantê-lo e outros não. Foi ainda avaliado o impacto das alterações associadas ao estilo de vida e hábitos alimentares após a cirurgia.

\section{Amostra e Métodos}

\subsection{Amostra}

O Centro Hospitalar de Entre o Douro e Vouga, E.P.E., com sede em Santa Maria da Feira, foi criado na sequência da publicação do Decreto-Lei n. 27/2009, de 27 de janeiro, agrupando o Hospital de São Sebastião', E.P.E. (Santa Maria da Feira), o Hospital Distrital de São João da Madeira e o Hospital São Miguel (Oliveira de Azeméis). A recolha de dados foi autorizada pelo médico cirurgião Doutor Mário Nora e teve a duração de três meses. O grupo de pacientes da consulta de obesidade do Hospital de São Sebastião

\footnotetext{
1 http://www.hospitalfeira.min-saude.pt, acedido a 01-06-2011.
} 
é acompanhado por uma equipa multidisciplinar, constituída por médicos, cirurgiões, psicólogos e nutricionistas. No âmbito do acompanhamento dos pacientes, são realizadas reuniões com a finalidade de esclarecer todo o processo cirúrgico e modificações daí resultantes.

Para este estudo, foram analisados 120 processos. Destes, 38 não foram considerados válidos, devido à ausência de informação final, tendo a análise e tratamento de dados incidido nos 82 processos válidos. Quando os processos foram analisados, recolheram-se informações sobre a idade, o sexo, o estado civil, a filiação, a profissão, o peso, a estatura, o índice de massa corporal, os antecedentes patológicos, fisiológicos e familiares, o tipo de cirurgia realizada, o grau de significância na perda de peso, assim como as alterações a nível da dieta alimentar e estilo de vida (Tabela 1). No presente estudo, os processos examinados incluíram unicamente pacientes operados há mais de três anos, dado que, segundo a opinião médica especializada, somente após este período é possível apurar um nível de sucesso significativo e estável relativamente à manutenção do peso perdido.

\subsection{Métodos}

No estudo retrospetivo, a metodologia empregue foi a análise de processos, para a qual foi estruturada uma ficha técnica do paciente com o propósito de recoIher todas as informações relevantes para o estudo em questão. A recolha destes dados teve por objetivo analisar a perda de peso após a intervenção cirúrgica em indivíduos obesos, verificar o seu grau de efetividade e tentar perceber a razão pela qual alguns pacientes conseguem perder peso e mantê-lo e outros não. A variável de sucesso estudada concentrou-se na eficácia da cirurgia bariátrica associada à prática de exercício físico e alterações do comportamento alimentar. Com a autorização por parte dos distintos profissionais, foi possível assistir a variadas reuniões informativas e educativas dirigidas aos pacientes. Assistiu-se à reunião pré-operatória cirúrgica e de nutrição (realizada à quarta-feira) e a reuniões psicoeducativas, efetuadas em seis sessões quinzenais (realizadas à segunda-feira). Relativamente ao grupo de apoio psicoterapêutico, não foi possível intervir na qualidade de observador, de forma a não expor os pacientes.

\subsubsection{Tratamento de dados}

Os dados obtidos foram introduzidos numa base de dados informatizada, utilizando o programa SPSS (Statistical Package for Social Sciences) versão 18.0. Para cada paciente, foram inseridos dados pessoais, patológicos, referências 
relacionadas com o peso inicial e final, com o tipo de cirurgia e as possíveis alterações dos hábitos alimentares e da atividade física e sua duração.

Foi realizada uma abordagem em que a variável dependente (a que chamamos também de sucesso) foi a diferença entre o IMC $C_{\text {inicial }}$ e o IMC final Esta estratégia permite expressar o sucesso com uma variável numérica. Quanto maior o valor obtido, maior a perda de massa corporal, por isso, maior o sucesso. A variável exercício físico também foi recodificada e foi considerada na sua vertente tempo de exercício com três níveis (nenhum exercício vs. $\leq 30 \mathrm{mi}-$ nutos vs.> 30 minutos). As diferenças entre as médias do sucesso foram testadas pelo teste estatístico ANOVA (Hair et al., 1995). Foi testada a igualdade de variâncias (homocedasticidade) com o teste de Levene. Em caso de heterocedasticidade foi considerada a correção de Brown-Forsythe. Os testes post hoc foram efetuados com o Sheffé test (se existia homocedasticidade) ou com o Howell (se existia heterocedasticidade). A profissão dos pacientes foi classificada segundo os critérios da Classificação Nacional de Profissões.

\section{Resultados}

Os antecedentes patológicos com valores mais relevantes no grupo de estudo foram as doenças cardiovascu- lares $(43 \%)$ e varizes $(13,9 \%)$, todavia o número de pacientes sem antecedentes patológicos foi igualmente significativo $(17,7 \%)$. Os valores menos relevantes estão relacionados com a doença da tiroide $(3,8 \%)$ e a anemia $(1,3 \%)$.

Comparativamente com os antecedentes fisiológicos, constatou-se na população observada uma grande disposição de indivíduos compulsivos (53,8\%), porém existe também uma elevada percentagem no que refere a sujeitos sem qualquer distúrbio alimentar (43,8\%). Os sujeitos que não têm uma influência familiar direta relacionada com a obesidade são em muito maior número $(73,1 \%)$ do que aqueles que apresentam antecedentes familiares de obesidade (26,7\%). O tipo de cirurgia mais frequente na população estudada foi o bypass (54,9\%), seguido da BGA (24,4\%). Por sua vez, a conversão da banda gástrica ajustável (BGA) para bypass representou uma preferência menor (20,7\%).

O exercício físico mostrou-se relevante para explicar o sucesso, medido pela diferença entre $\mathrm{O} I \mathrm{MC}_{\text {inicial }}$ e $\mathrm{IMC_{ \text {fi- } }}$ nal $\left(F_{2,81}=14.048, p=.000\right)$. Os testes post hoc foram utilizados para identificar que entre níveis da variável a diferença observada foi estatisticamente significativa. O sucesso aumenta com o tempo de exercício, porém as diferenças entre os que fazem $\leq 30$ minutos de exercício e os que fazem > 30 minutos não foram estatisticamente significativas ( $p=.363)$. 
Tabela 1. Caracterização sociodemográfica e clínica da amostra

\begin{tabular}{|c|c|c|c|}
\hline & & $\mathrm{N}$ & $\%$ \\
\hline \multirow{3}{*}{ Sexo } & Masculino & 15 & 18,3 \\
\hline & Feminino & 67 & 81,7 \\
\hline & Total & 82 & 100,0 \\
\hline \multirow{6}{*}{ Idade } & $19-30$ & 13 & 15,9 \\
\hline & $31-40$ & 31 & 37,8 \\
\hline & $41-50$ & 23 & 28,0 \\
\hline & $51-60$ & 13 & 15,9 \\
\hline & $>60$ & 2 & 2,4 \\
\hline & Total & 82 & 100,0 \\
\hline \multirow{4}{*}{ Estado Civil } & Solteiro/a & 7 & 10,1 \\
\hline & Casado/a ou União de facto & 59 & 85,5 \\
\hline & Viúvo/a ou Divorciado/a & 3 & 4,4 \\
\hline & Total & 69 & 100,0 \\
\hline \multirow{5}{*}{$\begin{array}{l}\text { Classificação das } \\
\text { profissões }^{\text {b) }}\end{array}$} & Trabalhadores não manuais & 11 & 19,3 \\
\hline & Trabalhadores manuais & 39 & 68,4 \\
\hline & Reformados & 3 & 5,3 \\
\hline & Desempregados & 4 & 7,0 \\
\hline & Total & 57 & 100,0 \\
\hline \multirow{9}{*}{$\begin{array}{l}\text { Antecedentes } \\
\text { patológicos }\end{array}$} & Doenças cardiovasculares & 34 & 43,6 \\
\hline & Anemia & 1 & 1,3 \\
\hline & Varizes & 11 & 14,1 \\
\hline & Distúrbios respiratórios do sono & 5 & 6,4 \\
\hline & Doença osteoarticular & 5 & 6,4 \\
\hline & Doença da tiroide & 3 & 3,8 \\
\hline & Doenças do foro psiquiátrico & 5 & 6,4 \\
\hline & Sem antecedentes patológicos & 14 & 18,0 \\
\hline & Total & 78 & 100,0 \\
\hline \multirow{4}{*}{$\begin{array}{l}\text { Antecedentes } \\
\text { fisiológicos }\end{array}$} & Compulsão & 43 & 53,8 \\
\hline & Bulimia & 2 & 2,4 \\
\hline & Sem antecedentes fisiológicos & 35 & 43,8 \\
\hline & Total & 80 & 100,0 \\
\hline \multirow{3}{*}{$\begin{array}{l}\text { Antecedentes } \\
\text { familiarese) }\end{array}$} & Com antecedentes familiares & 21 & 26,9 \\
\hline & Sem antecedentes familiares & 57 & 73,1 \\
\hline & Total & 78 & 100,0 \\
\hline \multirow{4}{*}{$\begin{array}{l}\text { Tipo de cirurgia } \\
\text { efetuada }\end{array}$} & Banda gástrica ajustável & 20 & 24,4 \\
\hline & Bypass & 45 & 54,9 \\
\hline & Banda e converte para bypass & 17 & 20,7 \\
\hline & Total & 82 & 100,0 \\
\hline
\end{tabular}

a) Não responderam 13 sujeitos (15,9\%); b) Não responderam 25 sujeitos $(30,5 \%)$; c) Não responderam 4 sujeitos $(4.9 \%)$; d) Não responderam 2 sujeitos (2.4\%); e) Não responderam 4 sujeitos (4.9\%)
A média do sucesso foi significativamente inferior naqueles que não fazem qualquer exercício físico, tanto quando comparados com os que praticam $>30$ minutos ( $p=.000$ ) como com os que praticam $\leq 30$ minutos $(p=.010)$ (Tabela 2$)$.

Também a dieta teve uma influência significativa para explicar o sucesso $\left(F_{2,14.36}=8,221, p=.004\right)$. O sucesso aumenta com um maior cumprimento da dieta. Os que cumprem a dieta e aqueles que a cumprem com dificuldade não obtiveram diferenças estatisticamente significativas ( $p=136$ ) no sucesso. As médias daqueles que não fazem dieta foram significativamente inferiores aos que cumprem ( $p=.000$ ) e aos que cumprem com dificuldade ( $p=.002)$.

A interação entre as duas variáveis (exercício e dieta) não foi estudada por não existirem observações suficientes para os cálculos em todas as células. O único sujeito que apesar de fazer exercício físico não cumpre a dieta sai do padrão de resultados, apresentando um sucesso elevado. Provavelmente outras variáveis explicam esta exceção.

Em média, os níveis de sucesso mais elevados ocorrem entre os que praticam mais de 30 minutos de exercício físico. Todos cumprem a dieta mesmo que relatem dificuldade (Tabela 3). No geral, perderam peso e estabilizaram $81,3 \%$ dos pacientes, os restantes 18,7\%, após a perda de peso, aumentaram mais de 5 quilos. 
Tabela 2. Médias, desvio-padrão e ANOVA do sucesso (IMCinicial - IMCfinal) em função da prática de exercício físico e do cumprimento da dieta

\begin{tabular}{|c|c|c|c|c|c|c|}
\hline & $\mathrm{N}$ & Média & DP & Levene & $\mathrm{F}_{2,81}$ & $P$ \\
\hline \multicolumn{7}{|l|}{ Exercício físico } \\
\hline Nenhum & 25 & 8,30 & 5,92 & $0,402^{\mathrm{ns}}$ & 14,048 & .000 \\
\hline$\leq 30$ minutos & 19 & 13,44 & 5,28 & & & \\
\hline$>30$ minutos & 38 & 15,60 & 5,03 & & & \\
\hline Dieta & & & & & $\mathrm{F}_{2,14.36}{ }^{\mathrm{a}}$ & \\
\hline cumpre dieta alimentar & 30 & 15,24 & 4,91 & $0,652^{\text {ns }}$ & 8,221 & .004 \\
\hline cumpre, mas com dificuldade & 45 & 12,60 & 5,61 & & & \\
\hline não cumpre dieta alimentar & 7 & 4,45 & 7,53 & & & \\
\hline
\end{tabular}

${ }^{a}$ Com correção deBrown-Forsythe; ns não significativo.

Tabela 3. Distribuição do grau de cumprimento da dieta alimentar pelo tempo de duração do exercício e média do sucesso IMCinicial - IMCfinal

\begin{tabular}{lcccccccccccccc}
\hline & \multicolumn{2}{c}{$\begin{array}{c}\text { Cumpre dieta ali- } \\
\text { mentar }\end{array}$} & \multicolumn{4}{c}{$\begin{array}{c}\text { Cumpre, mas com } \\
\text { dificuldade }\end{array}$} & \multicolumn{3}{c}{$\begin{array}{c}\text { Não cumpre } \\
\text { dieta alimentar }\end{array}$} & \multicolumn{3}{c}{ Total } \\
\hline \multirow{3}{*}{ Nenhum } & N & M & DP & N & M & DP & N & M & DP & N & M & DP \\
$\leq 30$ minutos & 7 & 10,31 & 4,65 & 12 & 10,37 & 5,48 & 6 & 1,82 & 3,19 & 25 & 8,30 & 5,92 \\
$>30$ minutos & 15 & 15,93 & 3,98 & 10 & 10,76 & 4,99 & 1 & 20,20 & & 19 & 13,44 & 5,28 \\
Total & 30 & 15,18 & 4,04 & 23 & 14,57 & 5,42 & 0 & & & 38 & 15,60 & 5,03 \\
& & & 4,91 & 45 & 12,60 & 5,61 & 7 & 4,45 & 7,53 & 82 & 12,87 & 6,18 \\
\hline
\end{tabular}

\subsection{Testemunhos ${ }^{2}$}

Paciente D: 97 quilos, engordou após uma mastectomia. “Eu só queria estar na cama e só comia na cama e comia

\footnotetext{
2 Os pacientes não são identificados pelo nome por uma questão de confidencialidade.
}

bastante. Tenho problemas nos ossos e sinto-me triste com o excesso de peso, não me sinto bem com este corpo."

Paciente G: 130 quilos. "Só penso em comer, passo o dia a ver programas de culinária, os meus sonhos é com comer e comer desalmadamente. Desde sempre fui composta. Por vezes perguntam-me 
se estou grávida e eu respondo não, não estou, sou gorda. Eu levo na brincadeira mas magoa."

\section{Discussão e Conclusões}

$\mathrm{Na}$ investigação em questão, é possível constatar a elevada adesão por parte do sexo feminino para tratamento da obesidade, existindo diversos estudos que corroboram esta tendência (Maggard et al., 2005; Colossi et al., 2008; Hwang et al., 2009). Em Portugal, a taxa de obesidade é mais elevada no sexo masculino do que no sexo feminino (Carmo et al., 2008). É possível que as candidatas femininas estejam mais preocupadas com o seu bem-estar do que os homens. Atualmente, mais de metade da população portuguesa dos 18 aos 64 anos de idade tem excesso de peso ou sofre de obesidade (Carmo et al., 2008).

A procura por cirurgia bariátrica é mais frequente por parte de pessoas na faixa etária entre os 31 e os 40 anos. Segundo Carmo et al. (2008), nesta média de idades verifica-se uma maior prevalência de pessoas obesas. Destes pacientes, os que mais procuram o tratamento cirúrgico são na sua maioria casados ou vivem em união de facto.

Uma outra categoria a que se tem dado particular ênfase é a profissão dos pacientes, uma vez que a obesidade tem sido tendencialmente associada ao baixo nível de instrução e rendimento (Pories, 2008). Através da pesquisa foi possível verificar que a taxa de obesidade é superior em classes profissionais consideradas manuais.

Atualmente, as patologias associadas à obesidade encontram-se bem definidas (Castelnuovo e Simpson, 2011). No grupo de estudo, as enfermidades mais proeminentes eram as doenças cardiovasculares e as varizes. Porém, a literatura tem referenciado melhorias após a intervenção cirúrgica, nomeadamente quanto a alguns cancros, asma, doenças infeciosas, infertilidade, apneia do sono, colesterol, triglicerídeos e diabetes (Kral, 2006). Uma vantagem relevante da técnica bypass é o seu efeito terapêutico na diabetes mellitus tipo 2, em que uma parte significativa dos pacientes operados (82\%) consegue recuperar substancialmente deste distúrbio (Karmali et al., 2010; Kashyap et al., 2010). Apesar destes benefícios, os mecanismos implícitos no tratamento continuam por esclarecer (Pories, 2008). Através da presente investigação, é possível afirmar que a cirurgia aplicada por excelência foi o bypass. Quanto à BGA, trata-se de um procedimento restritivo que respeita a fisiologia digestiva e deste modo é menos agressiva, contudo requer uma mudança importante nos hábitos alimentares e a longo prazo não é eficaz (Lemos et al., 2005). Por outro lado, o bypass é considerado a técnica ideal e é uma das 
práticas mais concretizadas na Europa e Estados Unidos da América (Lemos et al., 2005). Outra área comum na investigação é o comportamento alimentar, já que muitos candidatos à cirurgia possuem um elevado nível de fome e baixos níveis de restrição alimentar (Hwang et al., 2009). Na população estudada, os distúrbios alimentares mais influentes são a compulsão alimentar, que representa mais de metade da amostra observada. A compulsão alimentar associada à obesidade foi descrita pela primeira vez em 1959, mas só recentemente foi classificada como uma desordem específica e ocorre quando um indivíduo ingere uma quantidade enorme de comida, com perda de controlo, pelo menos duas vezes por semana durante seis meses (Jebb, 1997). Relativamente ao cumprimento da dieta alimentar, a maioria dos pacientes cumpriu a dieta, ainda que uma grande parte tenha assumido dificuldade na execução do plano alimentar. Contudo, o desempenho positivo dos pacientes quanto à dieta alimentar parece estar associado efetivamente à perda de peso. Em contrapartida, uma parte dos que não cumpriram o plano nutritivo voltou a ganhar peso.

Porém, a alimentação não foi a única alteração observada na vida dos pacientes, a prática de exercício físico foi importante para explicar o sucesso. A atividade física tem sido apontada como uma ferramenta fundamental na perda de peso posteriormente à cirurgia bariátrica. A maioria dos estudos conclui que os pacientes ativos perdem mais peso do que aqueles que continuam a levar uma vida sedentária após a cirurgia (Livhits et al., 2010). Indubitavelmente, a obesidade está relacionada com uma vida sedentária (Kaila e Raman, 2008). O exercício físico ajuda a prevenir o excesso de massa gorda e traz consigo benefícios físicos e psicológicos (Nunes, 2006). Para além dos hábitos alimentares e da atividade física, existem outros fatores que influenciam a perda de peso, como, por exemplo: a idade, o sexo, a composição corporal, a educação, o estado emocional e a etnia (Pories, 2008). Todavia, a probabilidade de voltar a ganhar peso é uma realidade e as razões que provocam esse novo agravamento do peso podem ser várias. Entre estas, encontram-se fatores como a ingestão de comidas muito calóricas, comer menos de cinco vezes ao dia, a desistência da prática de exercício físico, idade avançada ou alterações na saúde mental e física que possam afetar o estilo de vida (Karmali et al., 2010). Um estudo com candidatos à cirurgia bariátrica apurou as razões implícitas na dificuldade em perder peso (Hwang et al., 2009). Estes indivíduos frequentemente citavam motivos relacionados com a dieta, a atividade física, a motivação, o autocontrolo e a força de vontade (Hwang et al., 2009). Raramente os candidatos relatavam fatores como a disponibilidade, custos, suporte social, ambiente físico ou conhecimento (Hwang et al., 2009). 
Enquanto os homens associam a obesidade a questões médicas, as muIheres, por sua vez, assumem as dificuldades que sentem em controlar os hábitos alimentares e atribuem o excesso de peso à elevada ingestão de alimentos (Hwang et al., 2009). Um estudo realizado recentemente identificou dois fatores importantes relacionados com o ganho de peso: o abuso sexual na infância e ser casado com um indivíduo alcoólico (Felitti et al., 2010). Contudo, são necessários mais estudos para averiguar outros agentes relacionados com o aumento de peso após a cirurgia bariátrica.

Com o trabalho etnográfico, foi possível verificar uma manifesta preocupação com a saúde em geral por parte dos pacientes, que desejavam restabelecer a sua qualidade de vida após a operação. $\mathrm{Na}$ sua maioria, associavam a obesidade ao excesso de comida, que era muitas vezes tida como um vício a ser superado. Porém, alguns exemplos indicam que nem sempre assumiram esta associação. Por exemplo, uma das pacientes referiu o seguinte: "eu dizia que não comia muito, sou sozinha com os meus meninos que são pequeninos, agora vejo realmente que fazia panelas enormes cheias de comida e nunca sobrava nada, é típico dizer que nunca comemos muito, mas na realidade a comida desaparecia e só podia ser eu."

Para além da preocupação com a saúde física, era percetível um mal-estar relacionado com a componente corpo- ral na maioria das pacientes. Grande parte destas não se sentia bem com o seu corpo, chegando mesmo a dizer que "só de pensar que vou começar a trabalhar e vou ter que ir experimentar roupa para o trabalho, tenho medo que não tenham o meu número". Nas reuniões era visível como algumas pacientes estavam motivadas em mudar e como acreditavam nos benefícios da cirurgia. Já as pacientes que se mostraram menos motivadas não voltaram às reuniões psicoeducativas. Nas mudanças relacionadas com a atividade física, as duas pacientes que se encontravam em fase de recuperação confirmaram como o exercício físico beneficiava a recuperação: "eu vou ao ginásio todos os dias e como podem ver as minhas pernas estão tonificadas"; "eu não vou ao ginásio, mas caminho todos os dias e, olhem, não estou nada mal". As pacientes que deram o seu testemunho nas reuniões psicoeducativas estavam a cumprir o plano alimentar sem grande dificuldade, demonstravam gosto pelo exercício físico e estavam visivelmente satisfeitas. Contudo, uma delas fez questão de afirmar que a cirurgia traz benefícios e também dificuldades a ultrapassar, dado que, no seu caso, passou pela experiência de perder 40 quilos num mês, o que interferiu com o seu equilíbrio.

De um modo geral, o sucesso na perda de peso aumenta com um maior cumprimento da dieta, mesmo quando esta é seguida com dificuldade. Por outro lado, os sujeitos que não praticam 
exercício físico e não cumprem a dieta têm uma menor probabilidade de atingir o sucesso relativamente à perda de peso significativa. Importa referir que um indivíduo conseguiu perder e manter o peso apesar de não cumprir a dieta, embora praticasse exercício físico. Provavelmente existirão outras variáveis para explicar esta ocorrência. É concludente que, de uma forma regular, os níveis de sucesso mais elevados ocorrem entre os que cumprem a dieta alimentar e que fazem exercício físico. As alterações da dieta e do estilo de vida não devem abranger somente os indivíduos operados, visto que atualmente existe uma nítida preocupação com a elevada taxa de obesidade observada nas crianças em Portugal, o que sugere que a próxima geração vai enfrentar um agravamento nos problemas de saúde (Carmo et al., 2008).

Uma resolução para a diminuição de peso é sem dúvida a cirurgia bariátrica, que tem crescido em popularidade nos últimos anos (Sarwer et al., 2010). Trata-se de um instrumento importante na perda de peso maciça, que provoca uma acentuada melhoria na vida do indivíduo nas mais diversas áreas. Para um melhor cuidado na saúde do paciente após a cirurgia bariátrica, é importante um acompanhamento multidisciplinar nos primeiros anos e este deve ser cumprido de modo exemplar por parte do paciente.

Nos primeiros tempos após a cirurgia, alguns pacientes demonstram insa- tisfação corporal associada ao excesso de pele visível nas várias zonas do corpo (abdominal, peito, braços) devido à acentuada perda de peso. Teoricamente, este descontentamento corporal tem um papel motivacional na procura da cirurgia plástica (Sarwer et al., 2010). No entanto, não é nítido se a cirurgia plástica leva a uma melhoria da imagem corporal, visto que o corpo sofre alterações, como por exemplo cicatrizes que implicam um novo ajustamento por parte do paciente (Sarwer et al., 2010). São necessários estudos adicionais de modo a aprofundar este tema tão contemporâneo. Se, de um modo geral, os pacientes desejam muito perder peso, em contrapartida experienciam um medo inconsciente relacionado com mudanças significativas devido à elevada perda de peso (Felitti et al., 2010). Concludentemente, a cirurgia é uma técnica que permite a perda de peso de uma forma visível e célere, porém, a dieta e o exercício físico são um componente de tratamento adicional para as pessoas obesas, já que o sucesso a longo prazo implica uma alteração efetiva de ambos (Nour, 2010).

O presente trabalho realça a preocupação por parte da antropologia médica em compreender e em responder a questões relacionadas com a obesidade, os tratamentos implícitos e os seus problemas e como estes influenciam e moldam a organização social, a cultura e o contexto humano. 


\section{Referências bibliográficas}

Brown, P. 1998. Understanding and applying medical anthropology. Londres, Mayfield.

Carmo, I.; Santos, O.; Camolas, J.; Vieira, J.; Carreira, M.; Medina, L.; Reis, L.; Myatt, J.; Galvão-Teles, A. 2008. Overweight and obesity in Portugal: national prevalence in 2003-2005. Obesity reviews, 9(1): 11-19. DOI: 10.1111/j.1467-789X.2007.00422.x.

Castelnuovo, G.; Simpson, S. 2011. Ebesity - E-health for obesity - new technologies for the treatment of Obesity in clinical psychology and medicine. Clinical Practice \& Epidemiology in Mental Health, 7: 5-8. DOI: 10.2174/1745017901107010005.

Colossi, F.; Casagrande, D.; Chatkin, R.; Moretto, M.; Barhouch, A.; Repetto, G.; Padoin, A.; Mottin, C. 2008. Need for multivitamin use in the postoperative period of gastric bypass. Obesity Surgery, 18(2): 187-191. DOI: 10.1007/ s11695-007-9384-8.

Cordain, L. 2002. The paleo diet: lose weight and get healthy by eating the food you were designed to eat. Nova Jersey, John Wiley \& Sons.

Felliti, V. J.; Jakstis, K.; Pepper, V.; Ray, A. 2010. Obesity: Problem, Solution, or Both? The Permanente Journal, 14(1): 24-31. DOI: 10.7812/TPP/09-107.

Garcia-Closas, R.; Berenguer; González, C. A. 2006. Changes in food supply in Mediterranean countries from 1961 to 2001. Public Health Nutrition, 9(1): 5360. DOI: 10.1079/PHN2005757.
Gilman, S. 2010. Obesity the biography. Nova Iorque, Oxford University Press.

Hair, J.; Anderson, R. E.; Tatham, R. L. 1995. Multivariate data: Analysis with readings. New Jersey, Prentice-Hall.

Hussain, A.; Mahmood, H.; El-Hasani, S. 2009. Can Roux-en-Y gastric bypass provide a lifelong solution for diabetes mellitus? Canadian Journal of Surgery, 52(6): 269-275.

Hwang, K.; Childs, J.; Goodrick, G. K.; Aboughali, W. A.; Thomas, E. J.; Johnson, C. W.; Yu, S. C.; Bernstam, E. V. 2009. Explanations for Unsuccessful Weight Loss Among Bariatric Surgery Candidate. Obesity Surgery, 19(10): 1377-1383. DOI: 10.1007/s11695-008-9573-0.

Jebb, S. 1997. Aetiology of obesity. British Medical Bulletin, 53(2): 264-285. Disponível em: http://bmb.oxfordjournals. org/content/53/2/264.short.

Kaila, B.; Raman, M. 2008. Obesity: A review of pathogenesis and management strategies. The Canadian Journal of Gastroenterology, 22(1): 61-68. Disponível em: http://www.ncbi.nlm.nih.gov/pmc/ articles/PMC2659122.

Karmali, S.; Stoklossa, C. J.; Sharma, A.; Stadnyk, J.; Christiansen, S.; Cottreau, D.; Birch, D. W. 2010. Bariatric surgery: A primer. Canadian Family Physician, 56: 873879. Disponível em: http://www.cfp. ca/content/56/9/873.long.

Kashyap, S. R.; Gatmaitan, P.; Brethauer, S.; Schauer, P. 2010. Bariatric surgery for type 2 diabetes: Weighing the impact 
for obese patients. Cleveland Clinic Journal Medicine, 77(7): 468-476. DOI: 10.3949/ccjm.77a.09135.

Kral, J. G. 2006. ABC of obesity-Management: Part III-Surgery. British Medical Journal, 333(7574): 900-903. DOl: 10.1136/ bmj.39035.494560.68.

Lemos, S.; Domingos, T.; Vinha, J.; Nadai, A.; Vasconcellos, C.; Ferragut, C. 2005. Nova Proposta de Tratamento Cirúrgico da Obesidade: Gastrectomia Vertical e Bypass Intestinal Parcial. Resultados Preliminares. Revista Brasileira de Videocirurgia, 3(3): 131-142. Disponível em: http://www.sobracil.org.br/revista/rv030303/artigo03.htm.

Livhits, M.; Mercado, C.; Yermilov, l.; Parikh, J. A.; Dutson, E.; Mehran, A.; Ko, C. Y.; Gibbons, M. 2010. Exercise Following Bariatric Surgery: Systematic Review. Obesity Surgery, 20(5): 657-665. DOI: 10.1007/s11695-010-0096-0.

Maggard, M.; Shugarman, L.; Suttorp, M.; Maglione, M.; Sugerman, H.; Livingston, E:; Nguyen, N.; Zhaoping, L.; Mojica, W:; Hilton, L.; Rhodes, S.; Morton, S.; Shekelle, P. 2005. Meta-analysis: surgical treatment of obesity. Annals of Internal Medicine, 142(7): 547-559. DOI: 10.7326/0003-4819142-7-200504050-00013.

Mintz, S. W. 2001. Comida e Antropologia: Uma breve revisão. Revista Brasileira de Ciências Sociais, 16(47): 31-42. DOI: 10.1590/S0102-69092001000300002.

Nour, N. 2010. Obesity in Resource-Poor Nations. Reviews in Obstetrics and Gyne- cology, 3(4): 180-184. Disponível em: http://www.ncbi.nlm.nih.gov/pmc/ articles/PMC3046736.

Nunes, L. 2006. A prescrição da actividade física. Lisboa, Editorial Caminho.

OMS - Organização Mundial de Saúde. 2014. 10 facts on obesity. [Online]. [Geneva], World Health Organization. [Acedido em 23-02-2015]. Disponível em: http://www.who.int/features/factfiles/obesity/en.

OMS - Organização Mundial de Saúde. 2015. Obesity and overweight. Fact sheet No 311. [Online]. [Geneva], World Health Organization. [Acedido em 23-02-2015]. Disponível em: http://www.who.int/ mediacentre/factsheets/fs311/en.

Padez, C. 1999/2000. Uma perspectiva antropológica da obesidade. Antropologia Portuguesa, 16-17:145-160.

Padez, C. 2002. Actividade física, obesidade e saúde: uma perspectiva evolutiva. Revista Portuguesa de Saúde Pública, 20(1): 11-20. Disponível em: http://hdl. handle.net/10316/13598.

Papelbaum, M.; Moreira, R. O. ;Gaya, C.; Preissler, C.; Coutinho, W. 2010. Impacto do índice de massa corporal no perfil psicopatológico de mulheres obesas. Revista Brasileira de Psiquiatria, 32(1): 42-46. DOl: 10.1590/S151644462010000100009.

Pinheiro, K. A. P. N. 2005. História dos hábitos alimentares ocidentais. Universitas: Ciências da Saúde, 3(1): 173-190. DOI: 10.5102/ucs.v3i1.553. 
Pories, W. J. 2008. Bariatric Surgery: Risks and Rewards. The Journal of Clinical Endocrinology and Metabolism, 93(11 Suppl 1): 89-96. DOI: 10.1210/jc.2008-1641.

Power, M.; Shulkin, J. 2009. The evolution of obesity. Baltimore, The Johns Hopkins University Press.

Sarwer, D. B.; Fabricatore, A. N. 2008. Psychiatric Considerations of the Massive Weight Loss Patient. Clinics in Plastic Surgery, 35(1): 1-10. DOI: 10.1016/j. cps.2007.08.006.

Sarwer, D. B.; Wadden, T. A.; Moore, R. H.; Eisenberg, M. H.; Raper, S. E.; Williams, N. 2010. Changes in Quality of Life and Body Image Following Gastric Bypass Surgery. Surgery for Obesity and Related Diseases: official journal of the American Society for Bariatric Surgery, 6(6): 608614. DOl: 10.1016/j.soard.2010.07.015.

Stearns, P. 2002 [1997]. Fat history: bodies and beauty in modern west. Nova lorque, New York UniversityPress.

Sites consultados

http://www.who.int/mediacentre/factsheets/fs311/en/ [acedido a 05-05-2011].

http://www.hospitalfeira.min-saude.pt [acedido a 01-06-2011].

http://www.iefp.pt/formacao/CNP/Paginas/CNP.aspx [acedido a 09-06-2011]. 